\title{
Intelligent Fault Diagnosis of Synchromesh Gearbox Using Fusion of Vibration and Acoustic Emission Signals for Performance Enhancement
}

\author{
T Praveenkumar ${ }^{1, *}$, M Saimurugan $^{2}$, K I Ramachandran ${ }^{2}$ \\ ${ }^{1}$ Department of Automobile Engineering, SRM Institute of Science and Technology, SRM Nagar, Kattankulathur, Tamilnadu- \\ 603203, India. \\ ${ }^{2}$ Department of Mechanical Engineering, Amrita School of Engineering, Coimbatore, Amrita Vishwa Vidyapeetham, India. \\ praveent.mtp@gmail.com
}

\begin{abstract}
Condition monitoring system monitors the system degradation and it identifies common failure modes. Several sensor signals are available for monitoring the changes in system components. Vibration signal is one of the most extensively used technique for monitoring rotating components as it identifies faults before the system fails. Early fault detection is the significant factor for condition monitoring, where Acoustic Emission (AE) sensor signals have been applied for early fault detection due to their high sensitivity and high frequency. In this paper, vibration and acoustic emission signals are acquired under various simulated gear and bearing fault conditions from the synchromesh gearbox. Then the statistical features are extracted from vibration and $\mathrm{AE}$ signals and then the prominent features are selected using J48 decision tree algorithm respectively. The best features from the vibration and $\mathrm{AE}$ signals are then fused using feature-level fusion strategy and it is classified using Support Vector Machine (SVM) and Proximal Support Vector Machine (PSVM) classifiers and it is compared with individual signals for fault diagnosis of the synchromesh gearbox. From the experiments, it is observed that the performance of the fault diagnosis system has been improved for the proposed feature level fusion technique compared to the performance of unfused vibration and $\mathrm{AE}$ feature sets.
\end{abstract}

Keywords: Fault diagnosis; Vibration signal; Acoustic Emission signal; Feature level fusion.

T. Praveenkumar et al. This is an open-access article distributed under the terms of the Creative Commons Attribution 3.0 United States License, which permits unrestricted use, distribution, and reproduction in any medium, provided the original author and source are credited.

\section{INTRODUCTION}

Gearboxes play an important role in various industries such as aerospace, automotive and heavy industries. Gearboxes in certain machinery operate under tough working conditions. The major component in gearboxes like bearings and gears are prone to damages like fatigue and pitting due to its continuous operation. Misalignment and unbalance are the most common faults that occur in rotating components (Sanjay Taneja. (2013)). So, it is very important to identify faults at the right time to prevent accidents. Proper maintenance ensures fast-paced production and it prevents monetary losses. Fault diagnosis of a rotating component can be done by a standard technique called condition monitoring. The vibration signals monitoring approach is the widely used method in condition monitoring and fault diagnosis of rotating components as one of the nondestructive methods (Bostjan Dolenc et al. (2016), Xiao Li Zhang et al. (2015)). In recent years, acoustic emission (AE) signal has gained more attention from researchers in rotating machine fault diagnosis and detection. The research work reported (Ruoyu Li (2012), Yongzhi Qu et al. (2014)) that compared to vibration signal, AE signals have an advantage of high sensitivity indications near locations of faults and insensitive to structural resonance by mechanical background noise. AE signals are used in fault diagnosis of rotating machines such as gearbox failure, bearing failure, transmission failure, etc (Kuan Fang He et al. (2012)). AE has an ability in the detection of bearing fault and application of $\mathrm{AE}$ signal in fault detection of helicopter gearboxes (Faris Elasha et al. (2015)).

Earlier the machines were made to run for a long run until damages occur and were then analyzed. Later they began to monitor the machines periodically and rectify the faults. The person operating the machines on a long run can identify the fault by the sound and vibrations produced by the system. Then as time progressed the technological advancements 
made the technique easier and it helped in identifying the faults at an earlier stage. This enabled the fault to be rectified immediately without much delay and thereby saving money and resources. Sensors were used to identify any anomaly in the signals (for example vibrations), and the user will be alerted if there is any problem with the machine. The condition of inaccessible gear of the rotating machine can be continuously monitored with the help of vibration signals by placing a sensor close to the source point. The status of the machine can be identified by extracting the features from the processed signals.

The fusion of information from sensors with different physical characteristics enhances the understanding of condition of machine elements for planning and decisionmaking. Multi-sensor data fusion seeks to combine information from different sensors to obtain more inferences than can be derived from a single sensor. In recent years, the usage of multi-sensor data fusion method in fault diagnosis of the rotating machine was intensively used (Safizadeh \& Latifi (2014), Ashkan Moosavian et al. (2015), Otman Basir \& Xiao Hong Yuan (2007), Sultan Binsaeid et al. (2009)). The single sensor can never be reliable and precise and it might have many limitations. Data fusion is nothing but a combination of knowledge from several sources, as it provides better information i.e., more relevant information. The aim of multi-sensor data fusion technique is to lower the detection error and to increase the reliability (Loutas et al. (2011)).

Feature level fusion of vibration and acoustic emission signals is followed in this work. Fusion technique has been widely used in many areas such as fault diagnosis of motor (Gang Niu et al. (2007), Bo-Suk Yang \& Kwang Jin Kim (2006), Hong Fei Wang \& Jiang Ping Wang (2000)), image ((Hung-Chih Chiang et al. (2001), robotics (Abidi, 1992)), etc. The recent research reported (Meghdad Khazaee et al. (2014)) that fault classification accuracy of fused vibration and acoustic emission signals was increased by up to $10 \%$ when compared to single sensor mode using DempsterShafer evidence theory for fault diagnosis of planetary gearbox faults. The feature-level fusion technique for classification of handwritten numeral database and face image database provides effective recognition accuracy (Jian Yang et al. (2003)). The fusion of features from vibration and sound signals using decision tree feature selection technique has proved to be an effective method for fault diagnosis of rotating machinery (Saimurugan \& Ramprasad (2017)). The methodology flow diagram is shown clearly in Figure 1.

Visual inspection and physical assessment alone will no longer provide adequate information for identification of faults, but for faster and reliable identification we are in need of automated diagnosis procedure. Basically, fault diagnosis is carried out in three stages. Feature extraction is the first step, where irrelevant and redundant data's can be transformed into a reduced set of data and the extracted features can contain relevant information. The second step is the feature selection, where the best features are selected from the extracted features from the previous step and the final step is the fault classification or identification (Wei Li et al. (2015)).

Time-domain analysis, frequency domain analysis and timefrequency domain analysis are the digital signal analysis techniques. The research work reported (Junyan Yang et al. (2007), David Logan \& Joseph Mathew (1996)) that timedomain statistical feature is used for fault diagnosis of rolling element bearing, which results in improved performance using SVM classifier. FFT technique alone is not sufficient to analyze the frequency content of the defective bearing signal and it is less effective for inner race bearing defect, in such a case time-frequency analysis plays a major role (Yang et al. (2007), Rai \& Mohanty (2007)). Time-frequency domain investigates waveform signal in both time and frequency domain (Rubini \& Meneghetti (2001), Li et al. (2012), Lei et al. (2013)). Statistical features remain found to be a good applicant over histogram features for fault diagnosis of rotating machinery using a fusion of sound signals (Saimurugan \& Nithesh (2017)). All the features can contribute towards classification, but the best features have to be selected before classification. A decision tree is a widely used technique for feature selection (Saimurugan et al. (2011)). The reports (Saimurugan \& Ramprasad (2017), Saimurugan \& Nithesh (2017), Praveenkumar et al. (2018)) have proved the performance of a system increased by selecting the dominant features of vibration and sound signals using decision tree algorithm for fault diagnosis of rotating machinery. Support Vector Machine (SVM) and Artificial Neural Network (ANN) are the most popular feature classification technique for fault diagnosis of rotating machinery. ANN requires higher training data and time and it is difficult to interpret analytically, so the alternative of ANN is SVM (Wei Li et al. (2015)). SVM is based on statistical learning theory and its classification accuracy is better than ANN due to the principle of risk minimization (Samanta et al. (2003)). PSVM is designed as a system of linear equation, which results in lower computation time and it yields a result close to SVM (Saravanan et al. (2010)). Based on the literature review, it is concluded that there is an extensive scope in using SVM and PSVM classifiers for the fault diagnosis of the gearbox.

The workflow of the paper is as follows: 1 . The vibration and acoustic emission signals are captured for various simulated fault conditions at various speeds and loads. 2 . The statistical features were then extracted from the acquired vibration and acoustic emission signals. 3 . The best features were selected from both the vibration and acoustic emission signals using the C4.5 decision tree algorithm. 4. The selected features from both the signals were fused using feature-level fusion technique, providing common weight to 
both the signals. 5. Then the fused signals were classified using SVM and PSVM classifiers and their performance were compared with the unfused individual signals.

\section{EXPERIMENTAL STUDIES}

The methodology flow diagram is shown in Fig. 1. Experimental setup and the experimental procedures are discussed in the following subsections.

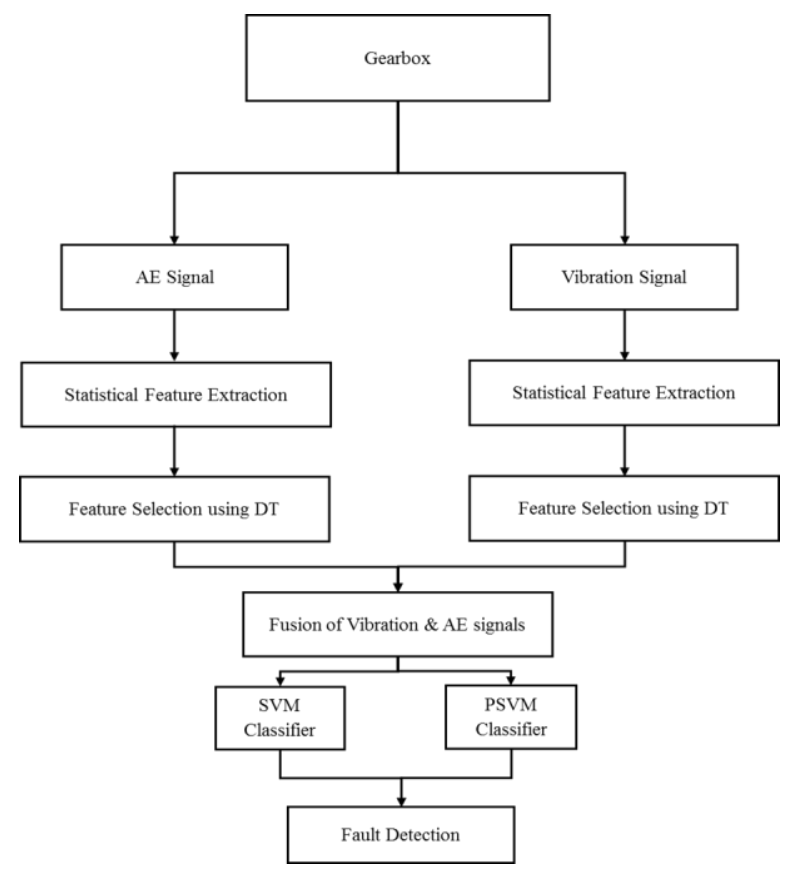

Figure 1. Methodology flow diagram

\subsection{Experimental Setup}

The automotive gearbox, motor, and dynamometer shown in Figure 2 form the experimental setup. The gearbox is driven by an AC motor. A control panel is used to adjust the speed of the motor from $50 \mathrm{rpm}$ to $1440 \mathrm{rpm}$. In this setup, the 4speed synchromesh automotive gearbox is used which can run at different gear speeds. Gear shifter serves the purpose of shifting gears in the gearbox and the top cover of the gearbox has been made flat to mount the sensor on it.

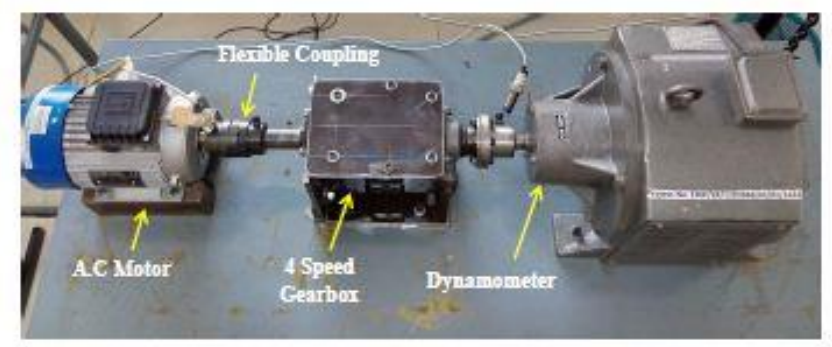

Figure 2. Experimental setup

All the systems are connected through flexible couplings which can act as a vibration damper, noise reducer and also matches some degree of misalignment between two shafts. Eddy current dynamometer and torque controller are employed to apply and vary loads respectively. Dytran triaxial accelerometer and acoustic emission sensor from physical acoustics were fixed on the top surface of the gearbox for acquisition of vibration and acoustic emission signals subsequently. The triaxial accelerometer is associated with $m+p$ Vibpilot Data Acquisition system and $\mathrm{AE}$ sensor are connected to a PCI based two-channel AE board, where the acquired analog signals has been converted into digital form and both the vibration and $\mathrm{AE}$ signals were stored separately.

\subsection{Experimental Procedure}

The experiment has been carried out using four different conditions. The initial condition is the good class where there is no fault induced in the gearbox setup. In the second condition, a fault was created on the outer race of the bearing using EDM (Electric Discharge Machining) and the gearbox is fitted with a faulty bearing. In the third condition, the face of the gear tooth was ground using a hand grinder to simulate the tooth damage. In the fourth condition, the gearbox is fitted with the faulty bearing and the gear with the damaged tooth. These conditions were analyzed for three different speeds and three different loading conditions. The twelve different fault conditions and their notations are mentioned from b1 to b12 in Table 1. All this trial was taken at three different motor speeds (500 rpm, $750 \mathrm{rpm}$, and $1000 \mathrm{rpm}$ ) for four different gear speeds and the data sets were acquired. The vibration and acoustic emission signals were acquired for a time period of one hundred seconds. Time-domain signals are sampled at $8.2 \mathrm{kHz}$ for vibration signals. Table 1 shows a 12-class problem for one particular motor and gearbox speed. For three motor speeds (500, 750 and $1000 \mathrm{rpm}$ ) and four gear speeds (1st, 2nd, 3rd, and 4th gear), the experiment has been carried out for 144 conditions. The time-domain plot of vibration signal for all 12 conditions at $500 \mathrm{rpm}$ is shown in Figure 3.

\begin{tabular}{lll}
\hline S.No & Parameter & \multicolumn{1}{c}{ Operating Conditions } \\
\hline i & b1 & $\begin{array}{l}\text { Good gear with good bearing and without } \\
\text { load }\end{array}$ \\
\hline ii & b2 & $\begin{array}{l}\text { Good gear with good bearing and load of } \\
5 N m\end{array}$ \\
\hline iii & b3 & $\begin{array}{l}\text { Good gear with good bearing and load of } \\
10 N m\end{array}$ \\
\hline iv & b4 & $\begin{array}{l}\text { Good gear with faulty bearing and } \\
\text { without load }\end{array}$ \\
\hline v & b5 & $\begin{array}{l}\text { Good gear with faulty bearing and load of } \\
5 N m\end{array}$ \\
\hline vi & b6 & $\begin{array}{l}\text { Good gear with faulty bearing and load of } \\
10 N m\end{array}$ \\
\hline vii & b7 & $\begin{array}{l}\text { Faulty gear with good bearing and } \\
\text { without load }\end{array}$ \\
\hline viii & b8 & $\begin{array}{l}\text { Faulty gear with good bearing and load of } \\
5 N m\end{array}$ \\
\hline
\end{tabular}




\begin{tabular}{lll}
\hline ix & b9 & $\begin{array}{l}\text { Faulty gear with good bearing and load of } \\
10 N m\end{array}$ \\
\hline $\mathrm{x}$ & b10 & $\begin{array}{l}\text { Faulty gear with faulty bearing and } \\
\text { without load }\end{array}$ \\
\hline xi & b11 & $\begin{array}{l}\text { Faulty gear with faulty bearing and load } \\
\text { of 5Nm }\end{array}$ \\
\hline xii & b12 & $\begin{array}{l}\text { Faulty gear with faulty bearing and load } \\
\text { of 10Nm }\end{array}$ \\
\hline
\end{tabular}

Table 1 Conditions analyzed in the experiment
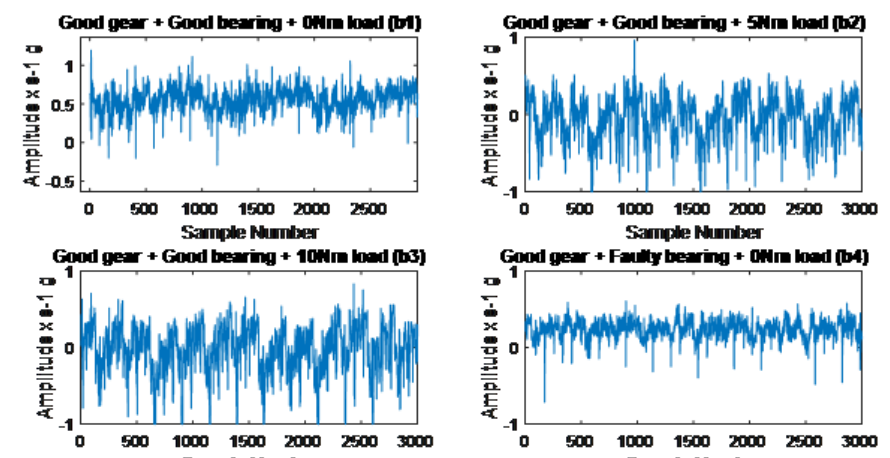

Sample Number

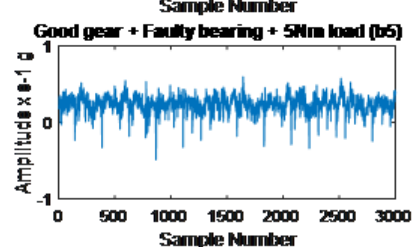

Sample Nunnber
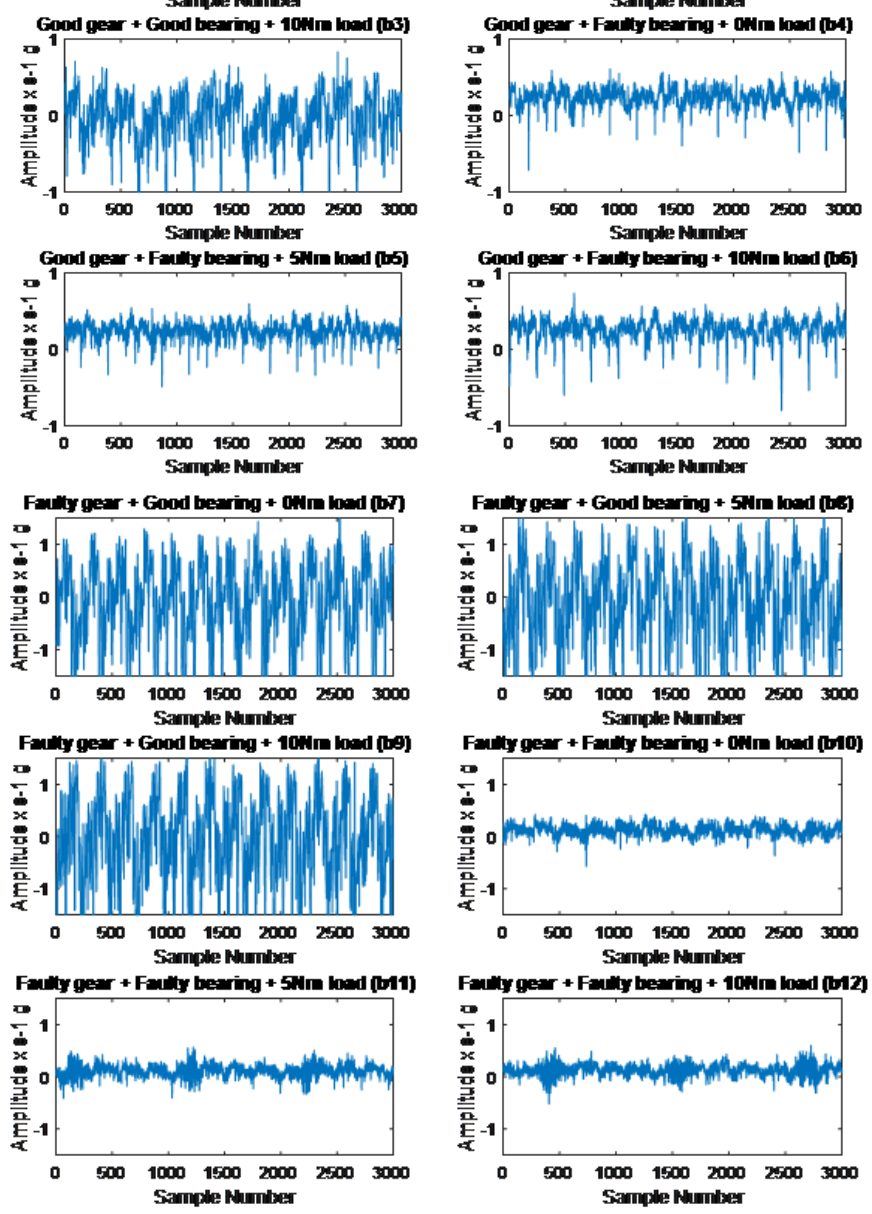

Figure 3.Time-domain plot of vibration signals

\section{FEATURE EXTRACTION}

\subsection{Statistical features of vibration signals}

Feature extraction is the dimensionality reduction technique in machine learning application extract features of faults. Vibration signals of a gearbox are usually non-stationary due to complex in structure. Hence the features of these signals can be extracted statistically to find the characters of vibration signals. Ten statistical features were used in this study and they are Mean, Median, Minimum, Mode, Maximum, Standard deviation, Sum, Kurtosis, Variance, and Skewness.

Mean: It generally deals with the sum of sampled value divided by a number of samples.

Sum: It is the sum of all the given data points.

Median: It shows a middle value in the sorted set of data.

Minimum value: It indicates a minimum data point value.

Maximum value: It indicates a maximum data point value.

Mode: It shows the most often appears value in a data set.

Standard deviation: It determines the actual energy of the vibration signal.

$$
\text { Standard deviation }=\sqrt{\frac{1}{N-1} \sum(x-\bar{x})^{2}}
$$

Variance: It is the measure of the distance of data points from mean.

Variance $=\frac{1}{N-1} \sum(x-\bar{x})^{2}$

Kurtosis: It presents the flatness or spikiness in the signal. For normal operating condition of the machine, it shows a flat signal and in faulty condition, it shows a spiky condition.

Kurtosis $=\left\{\frac{n(n+1)}{(n-1)(n-2)(n-3)} \sum\left(\frac{x-\bar{x}}{s}\right)^{4}\right\}-\frac{3(n-1)^{2}}{(n-2)(n-3)}$

Skewness: It measures the asymmetry of the distribution around its mean.

Skewness $=\frac{n}{n-1} \sum\left(\frac{x-\bar{x}}{s}\right)^{3}$

Where $\mathrm{x}$ is the sample,

$\mathrm{n}$ is the number of samples per second,

$\bar{x}$ is the mean,

$\mathrm{S}$ is the standard deviation.

\subsection{Statistical Features of Acoustic Emission Signals}

The Acoustic emission signal features considered in this study are rise time, count, energy, duration, amplitude, AFreq, RMS, Average Signal Level (ASL), Percentile, Thr, R-Freq, I-Freq, Signal strength, and Absolute energy and each of the AE features shown in Figure 4 is described below.

Rise time: It is the time to wait between the peak signal and principal threshold.

Counts: It refers to the number of threshold crossings.

Energy: Integral of the squared amplitude over the signal duration. 


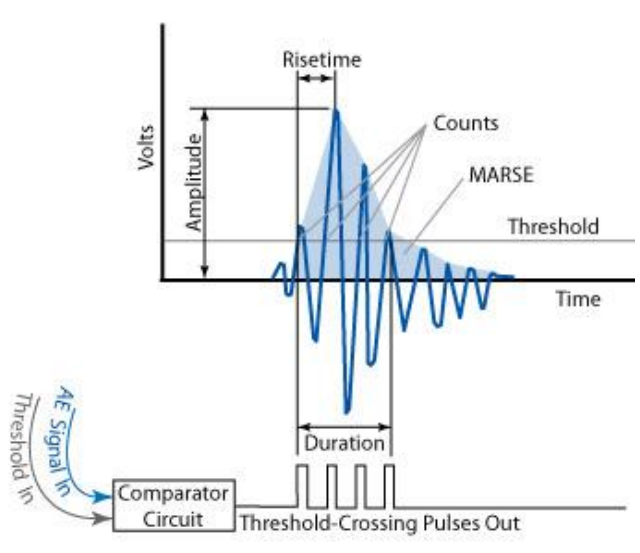

Figure 4. Features of AE signal

Duration: It is the time difference between the crossing of the first and last threshold.

Amplitude: It is the measure of the degree of change due to atmospheric pressure and is measured in decibels $(\mathrm{dB})$.

RMS: It is a measure of signal intensity

Average Signal Level (ASL): It defines the variation in signal amplitude and reported in $\mathrm{dB}$ unit.

Marse: It is referred to as energy counts

\section{Thr: Threshold limit}

Signal strength: Represent the area under the corrected signal envelope

Absolute energy: It is obtained from the integration of the squared voltage signal divided by a reference resistance over the duration of an acoustic emission

Based on the character of the material and the magnitude of the $\mathrm{AE}$ event, one hit can create one or many counts. It generally needs to be combined with amplitude or duration measurements to give valuable information about the shape of a signal.

\section{FEATURE SELECTION}

Feature selection plays a major role in machine learning application. Feature selection technique is used to reduce the input features to an acceptable size for further processing and it is also called a dimensionality reduction technique.

This is one of the data pre-processing techniques. In this work, the J48 decision tree algorithm is used for feature selection and it is the improved version of C4.5 algorithm. A decision tree is a simplest but powerful algorithm which generates IF-THEN rules and it forms a top-down induction method similar to trees such as root node, an intermediate node, and leaf node. The input to the decision tree is the statistical features that were extracted. The leaves in the decision tree represent the class labels. Each branch represents a possible value of the node from which it is originated. The most useful parameter for classification in a node can be selected using certain criteria using the concept of entropy and information gain. The decision tree algorithm involves 2 phases: building phase and pruning phase.

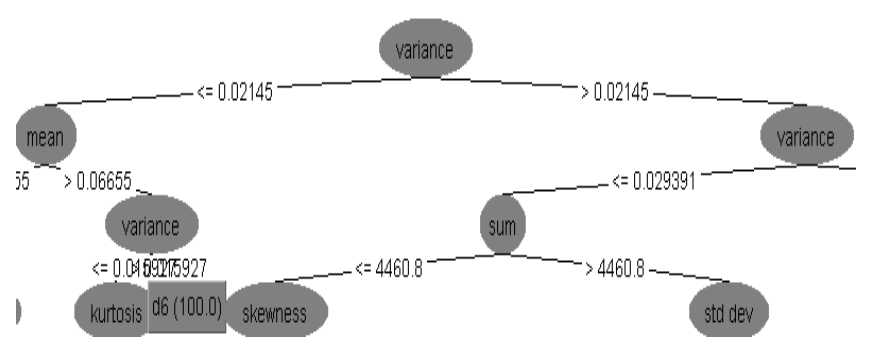

Figure 5. Decision tree-Classification of statistical features using vibration signal

The part of decision tree for 12 class problem with an operating condition of 3rd gear $500 \mathrm{rpm}$ using a vibration signal is shown in Figure 5. Figure 5 shows a part of the decision tree, where the top root node is the best node and the remaining nodes are arranged in a descending order based on its importance. Only the contributed features for classification will appear in the tree, so it can be used for selecting features which contribute more towards classification. It selects the best features from the training data set, thus reduces the process for pattern recognition. In Figure 5, the selected features are variance, mean and sum.

The output of the decision tree obtained in selecting statistical features of AE signal for 12 class problem with an operating condition of 3rd gear at $500 \mathrm{rpm}$ is shown in Figure 6. RMS, ASL, and Duration were the selected features for this particular operating condition.

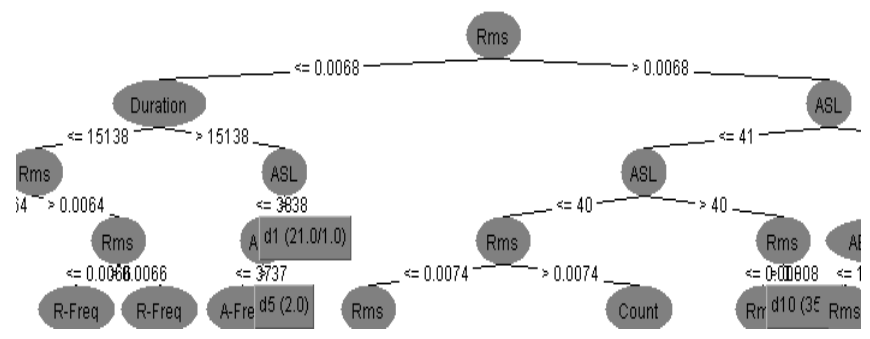

Figure 6. Decision tree-Classification of statistical features using $\mathrm{AE}$ signal

\section{SENSOR FUSION TECHNIQUE}

Data mining and machine learning are the techniques which infer knowledge from raw data and then analyze the data, which gives a way for automated interpretation of the sensor data and fault classification. While the sensor fusion technique combines data from multiple sources in order to get clear and complete information about the machine. These techniques would work collaboratively within the health assessment process of a Machine Condition 
Monitoring (MCM) system to allow for complete, tested and automatic interpretation of the raw data.

Sensor fusion technique is divided into three levels. These levels are:

1. Data level: Data level fusion is the basic technique, where the information from each sensor is combined directly. Then the features will be extracted and sent to the classifier as input. The limitation of data-level fusion is the sensor and data type to be necessarily equivalent.

2. Feature level: In this level, the features from each sensor are individually extracted and it is fused into a relative group which is used as an input to the classifier.

3. Decision level: In decision level fusion, the information from the sensors are extracted and classified separately. Then, the result from the classifiers is fused after each sensor. It is also called as classifier fusion.

Feature level fusion technique is employed in this work by combining the best-selected features of vibration and acoustic emission signals after the feature selection process.

\section{FEATURE CLASSIFICATION}

\subsection{Support Vector Machine}

SVM comes under a class of supervised learning algorithm, where a set of features has been given as input to the learning machine with desired output values (signals/labels). Each feature has been considered as a dimension of a hyperplane.

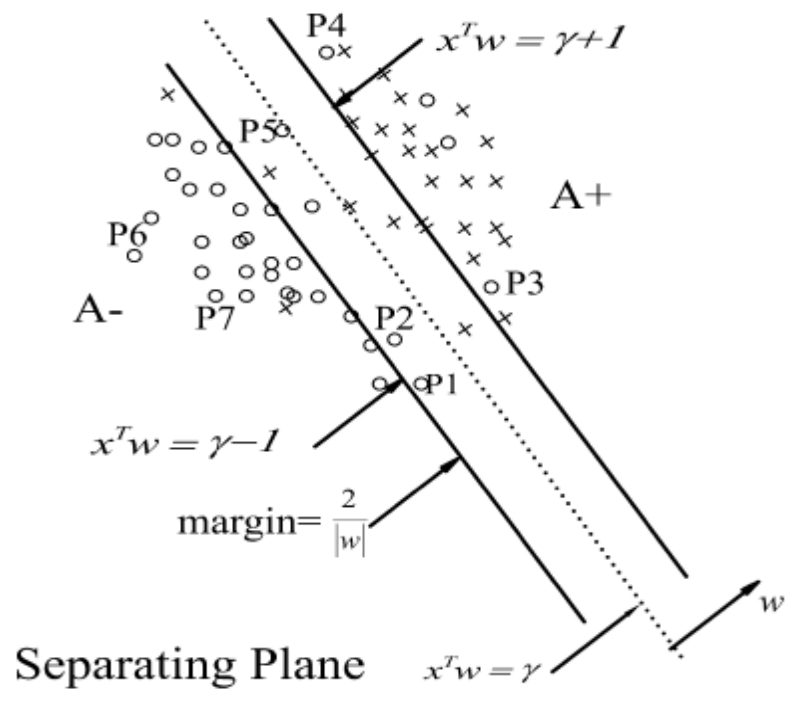

Figure 7. Standard SVM Classifier

Generally, SVM classifier constructs a hyperplane that separates the data point into two or multi-class problem is shown in figure 7. By doing this, the algorithm tries to increase the margin in order to reduce the generalization error. Generalization error deals that when a new feature is sent for classification, the error in prediction of class using learned knowledge will be minimal. This type of classifier will maintain a higher margin between the classes. Thus, maximizing the margin can make the hyperplane parallel to the bounding plane and the distance between these two planes are called as 'margin'. The data points on or near to the bonding planes are known as support vectors. The points P1 to P5 are called as support vectors and it belongs to class ' $\mathrm{A}^{+}$' and ' $\mathrm{A}$ ', but the points P6 and P7 far away from bounding planes and they are not support vectors. Support vectors play a major role in classification and hence it is called as support vector machines.

If the training features are split without any error by a hyperplane, then the error rate of testing features is based on the ratio of support vectors to the number of training vectors. The smaller support vectors will result in more general results and it is independent of the dimension of the problem.

\subsection{Proximal Support Vector Machine}

A simple and effective Proximal Support Vector Machine (PSVM) classifier is the developed version of SVM. In PSVM each point is assigned too close to the bonding planes and they have pushed apart as far as possible. This formation can lead to a simple and faster algorithm for solving a single system of a linear equation. The point of difference from SVM is given in the equation below.

$\min _{(w, \gamma, y)} v \frac{1}{2}\|y\|^{2}+\frac{1}{2}\left(w^{T} w+\gamma^{2}\right)$

s.t. $D(A w-e \gamma)+\mathrm{y}=\mathrm{e}$.

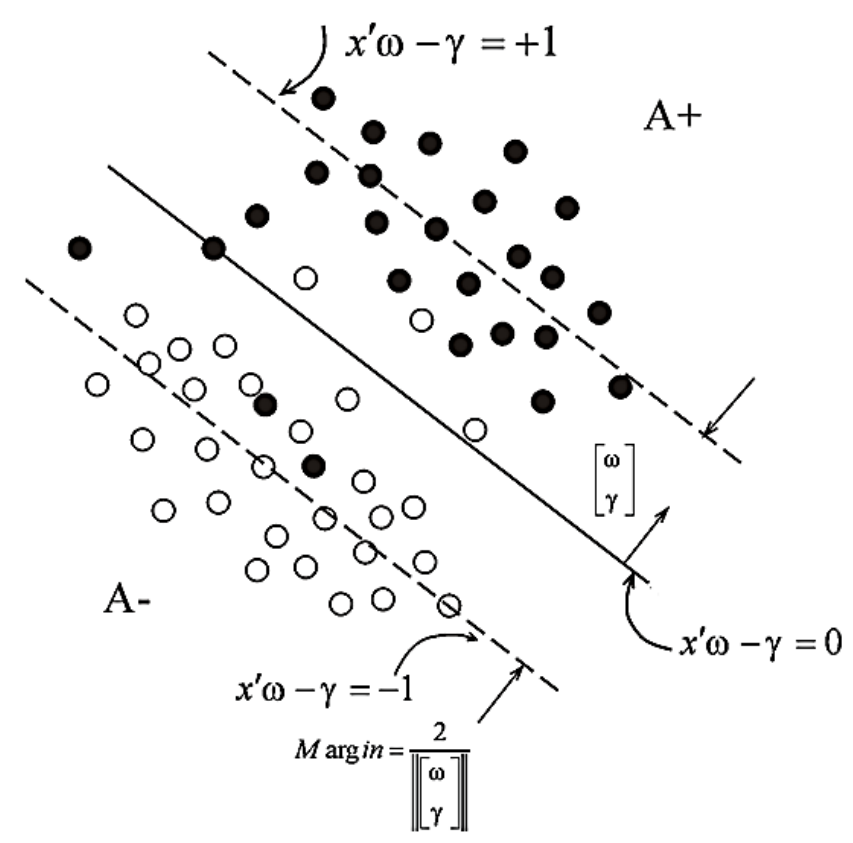

Figure 8. Proximal Support Vector Machine 
Figure. 8 shows the formulation of geometrical interpretation, where $y$ represents the deviation of the data points from a central plane to which it belongs to $(\mathrm{A}+$ or $\mathrm{A}$ ) .

The planes $w T w-\gamma= \pm 1$ are called as "proximal" planes, in which the points of each class are clustered and they are pushed as far as possible by the term $w T w+\gamma 2$ in the objective function; in the term is the reciprocal of the 2norm distance squared between the two planes $w, \gamma$. The idea is not based on maximizing the distance between the bounding parallel planes, which are the key feature of SVMs. After training, prediction of its class for any new set of features is possible using the decision function which is a function of ' $w$ ' and ' $\gamma$ ' as given below and it is called testing.

$$
f(x)=\operatorname{sign}(w T x-\gamma)
$$

If the sign value of $f(x)$ is positive then the new set of features belongs to class $\mathrm{A}+$, if sign value is negative then it belongs to class A-. Multiple class classification is commonly performed by combining several binary SVM classifiers.

\subsection{Application of SVM and PSVM for Fault Diagnosis for Automobile Gearbox}

For both gear and bearing fault classes, six features from both the vibration and acoustic emission signals consisting of 144 experimental conditions was collected for $500 \mathrm{rpm}$, $750 \mathrm{rpm}$, and $1000 \mathrm{rpm} .100$ samples were used in each fault classes, where 75 samples are used for training and 25 samples are engaged for testing. SVM model with radial basis function (RBF) kernel is used in this study, where research work reported (Saimurugan et al., 2011) SVM classifier with RBF kernel perform better than other kernel functions and it is the good candidate for fault diagnosis of rotating mechanical systems. The trained values of ' $w$ ' and ' $\gamma$ ' and its accuracies were tabulated in Table 2

\section{RESULTS AND DISCUSSION}

A better discriminating fault condition has been identified from statistical features for both vibration and acoustic emission features. In PSVM, the weight $(w)$ and gamma $(\gamma)$ define the separating plane. The value of weight and gamma is different for the same testing condition (for gear 1 at 500 $\mathrm{rpm}, 750 \mathrm{rpm}$, and $1000 \mathrm{rpm}$ ). As the speed changes, the corresponding magnitude of vibration changes and its weight and gamma will also change (Table 2) based on the speed and hence statistical parameter changes with speed.

\begin{tabular}{|c|c|c|c|c|}
\hline Gear & $\begin{array}{l}\text { Speed } \\
(\mathrm{rpm})\end{array}$ & $\begin{array}{l}\text { Weight } \\
(w)\end{array}$ & $\operatorname{Gamma}(\gamma)$ & $\begin{array}{l}\text { Classification } \\
\text { accuracy }(\%)\end{array}$ \\
\hline 1 & 500 & $\begin{array}{c}0.7673 \\
0.1754 \\
0.0110 \\
-0.0447\end{array}$ & -2.275 & 97.25 \\
\hline 2 & 500 & $\begin{array}{c}0.1425 \\
-0.0096 \\
0.6550 \\
-0.0017\end{array}$ & -3.29 & 96.71 \\
\hline 3 & 500 & $\begin{array}{l}-0.0000 \\
-0.0001 \\
-0.0057 \\
-0.0000\end{array}$ & -0.0127 & 98.69 \\
\hline 4 & 500 & $\begin{array}{c}-0.0397 \\
0.0146 \\
0.0031 \\
0.0416\end{array}$ & -0.0085 & 99.89 \\
\hline 1 & 750 & $\begin{array}{c}3.9052 \\
0.1056 \\
-0.5309 \\
0.0003\end{array}$ & -1.55 & 97.78 \\
\hline 2 & 750 & $\begin{array}{c}0.2135 \\
-0.0565 \\
-0.0272 \\
-0.0023\end{array}$ & -3.34 & 97.21 \\
\hline 3 & 750 & $\begin{array}{c}0.0481 \\
-0.0486 \\
0.0907 \\
-0.0035\end{array}$ & -0.8684 & 98.84 \\
\hline 4 & 750 & $\begin{array}{c}-0.0346 \\
-0.0375 \\
0.0088 \\
0.0423\end{array}$ & -0.0085 & 99.48 \\
\hline 1 & 1000 & $\begin{array}{c}0.7447 \\
-0.1106 \\
-1.1150 \\
-0.0006\end{array}$ & -2.80 & 98.17 \\
\hline 2 & 1000 & $\begin{array}{c}0.0300 \\
-0.0206 \\
0.1381 \\
0.0060\end{array}$ & -0.45 & 98.03 \\
\hline 3 & 1000 & $\begin{array}{c}-0.0385 \\
-0.0389 \\
0.0204 \\
-0.0006\end{array}$ & -0.0899 & 99.35 \\
\hline 4 & 1000 & $\begin{array}{c}0.0135 \\
-0.0117 \\
-0.3716 \\
0.2134\end{array}$ & -0.0223 & 98.84 \\
\hline
\end{tabular}

Table 2 PSVM performance for fused data set for three speeds

A decision tree represents the features that will have high importance in classification. Top three contributors from both the vibration and acoustic emission signal features were fused using a feature level fusion technique for further classification. The level of contribution from all the features is not equally important. The level of contribution from the individual statistical features is measured using a decision 
tree algorithm (fig.5). From figure 5, the top three contributors have been identified as dominant features namely variance, mean and sum for vibration signal at $3^{\text {rd }}$ gear $500 \mathrm{rpm}$ condition. In figure 6, RMS, duration, and ASL were selected as the dominant features for AE signals for the same operating condition. The selected top three features from vibration and acoustic emission signal were fused together and classified using SVM and PSVM classifier. Table 3 shows the classification accuracy of SVM for individual signal features and fused features.

The lower band accuracy of SVM with a vibration signal gives $79.50 \%$ whereas the upper band accuracy of $99.25 \%$. But the acoustic emission signal gives a lower band accuracy of $63 \%$ and upper band accuracy of $94.25 \%$. It shows that the vibration signal is highly effective than $\mathrm{AE}$ signal. But the classification accuracy of these two individual signals is not sufficient for identification of gearbox faults effectively. The fused signals of vibration and acoustic emission signals with SVM classifier provides a mean classification accuracy of $95.30 \%$, which is better compared to unfused individual signals. This result proves that the multi-sensor signal fusion with SVM classifier is more effective than the unfused individual signals.

The classification accuracy of gearbox faults using PSVM classifier is tabulated in Table 4. The obtained result shows that the minimum classification accuracy of vibration signal with PSVM classifier is $94.36 \%$ whereas the maximum classification accuracy of $99.54 \%$. The acoustic emission signal gives a minimum value of $92.41 \%$ and a maximum value of $98.02 \%$. The fused signals of vibration and acoustic emission signals with PSVM classifier delivers a mean classification accuracy of $98.35 \%$.

\begin{tabular}{|c|c|c|c|c|c|c|c|c|c|c|c|c|}
\hline \multicolumn{13}{|c|}{ SVM Classification accuracy } \\
\hline & \multicolumn{4}{|c|}{$500 \mathrm{rpm}$} & \multicolumn{4}{|c|}{$750 \mathrm{rpm}$} & \multicolumn{4}{|c|}{$1000 \mathrm{rpm}$} \\
\hline & $\begin{array}{c}1^{\text {st }} \\
\text { Gear }\end{array}$ & $\begin{array}{c}2^{\text {nd }} \\
\text { Gear }\end{array}$ & $\begin{array}{c}3^{\text {rd }} \\
\text { Gear }\end{array}$ & $\begin{array}{c}4^{\text {th }} \\
\text { Gear }\end{array}$ & $\begin{array}{c}1^{\text {st }} \\
\text { Gear }\end{array}$ & $\begin{array}{l}2^{\text {nd }} \\
\text { Gear }\end{array}$ & $\begin{array}{c}3^{\text {rd }} \\
\text { Gear }\end{array}$ & $\begin{array}{c}4^{\text {th }} \\
\text { Gear }\end{array}$ & $\begin{array}{c}1^{\text {st }} \\
\text { Gear }\end{array}$ & $\begin{array}{l}2^{\text {nd }} \\
\text { Gear }\end{array}$ & $\begin{array}{c}3^{\text {rd }} \\
\text { Gear }\end{array}$ & $\begin{array}{c}4^{\text {th }} \\
\text { Gear }\end{array}$ \\
\hline $\begin{array}{c}\text { Vibration } \\
\text { signal } \\
\end{array}$ & 88.56 & 92.06 & 96.00 & 98.50 & 90.56 & 87.19 & 96.83 & 93.35 & 79.50 & 89.19 & 99.25 & 93.33 \\
\hline $\begin{array}{c}\text { Acoustic } \\
\text { emission } \\
\text { signal }\end{array}$ & 66.19 & 63.00 & 83.58 & 91.08 & 77.69 & 83.19 & 90.83 & 94.25 & 88.50 & 91.00 & 92.33 & 84.42 \\
\hline Fused & 89.94 & 96.63 & 94.75 & 99.83 & 89.56 & 90.81 & 94.17 & 99.42 & 93.56 & 96.56 & 99.75 & 98.75 \\
\hline
\end{tabular}

\begin{tabular}{|c|c|c|c|c|c|c|c|c|c|c|c|c|}
\hline \multicolumn{13}{|c|}{ PSVM Classification accuracy } \\
\hline & \multicolumn{4}{|c|}{$500 \mathrm{rpm}$} & \multicolumn{4}{|c|}{$750 \mathrm{rpm}$} & \multicolumn{4}{|c|}{$1000 \mathrm{rpm}$} \\
\hline & $\begin{array}{c}1^{\text {st }} \\
\text { Gear }\end{array}$ & $\begin{array}{c}2^{\text {nd }} \\
\text { Gear }\end{array}$ & $\begin{array}{c}3^{\text {rd }} \\
\text { Gear }\end{array}$ & $\begin{array}{c}4^{\text {th }} \\
\text { Gear }\end{array}$ & $\begin{array}{c}1^{\text {st }} \\
\text { Gear }\end{array}$ & $\begin{array}{c}2^{\text {nd }} \\
\text { Gear }\end{array}$ & $\begin{array}{c}3^{\text {rd }} \\
\text { Gear }\end{array}$ & $\begin{array}{c}4^{\text {th }} \\
\text { Gear }\end{array}$ & $\begin{array}{c}1^{\text {st }} \\
\text { Gear }\end{array}$ & $\begin{array}{c}2^{\text {nd }} \\
\text { Gear }\end{array}$ & $\begin{array}{c}3^{\text {rd }} \\
\text { Gear }\end{array}$ & $\begin{array}{c}4^{\text {th }} \\
\text { Gear } \\
\end{array}$ \\
\hline $\begin{array}{c}\text { Vibration } \\
\text { signal } \\
\end{array}$ & 96.99 & 95.56 & 97.63 & 99.54 & 95.38 & 95.39 & 95.30 & 96.63 & 95.61 & 94.36 & 97.11 & 97.69 \\
\hline $\begin{array}{l}\text { Acoustic } \\
\text { emission } \\
\text { signal }\end{array}$ & 96.30 & 92.41 & 96.54 & 97.97 & 95.31 & 94.93 & 97.02 & 98.02 & 94.94 & 95.96 & 97.64 & 96.86 \\
\hline Fused & 97.25 & 96.71 & 98.69 & 99.89 & 97.78 & 97.21 & 98.84 & 99.48 & 98.17 & 98.03 & 99.35 & 98.84 \\
\hline
\end{tabular}




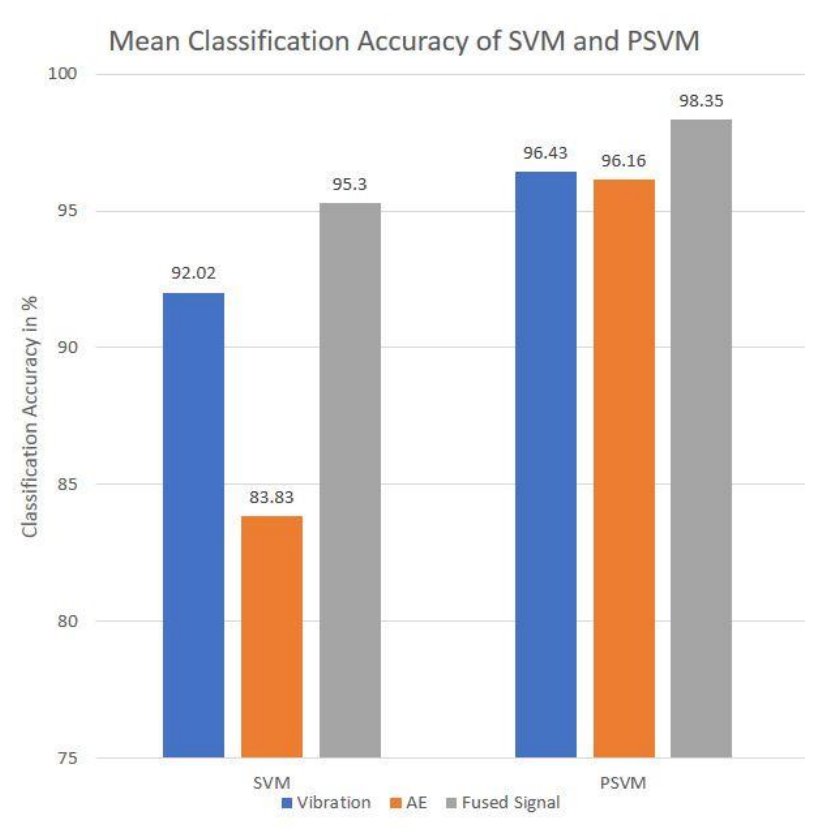

Figure 9. Mean Classification Accuracy using SVM and PSVM Classifiers

Figure 9 shows the validation of PSVM with SVM results, where the vibration signals are more effective than acoustic emission signals in both the classifiers. But PSVM performs better for both vibration as well as acoustic emission signals individually, and the mean classification accuracy of PSVM for the fused vibration and acoustic emission signals gives an accuracy of $98.35 \%$ which is quite better than unfused individual signals. Here also PSVM validates the multisensor fusion technique which enhances the performance of the classifier and it is shown in Figure 9.

The multi sensor information fusion based fault detection was discussed in detail. The present work proved that fusion of signals from multiple sensors yield better classification accuracy when compared to the individual signals. The classification accuracy of fused signals using SVM and PSVM provides a better result than individual vibration and acoustic emission signals. But the effective classifier like PSVM performs better for both fused as well as unfused individual signals. From the research work, it can be concluded that multi sensor data fusion with PSVM can be highly effective for gearbox fault diagnosis.

\section{CONCLUSION}

In this work, the time domain statistical features were extracted from the vibration and acoustic emission signals, and the selected best features from both the signals were fused for fault diagnosis of the synchromesh gearbox. Experiments were carried out at different speeds and loading conditions for various fault conditions. The prominent features were selected using the J48 decision tree algorithm, and then the faults are classified using SVM and PSVM classifiers. From these experiments, it is observed that SVM classifier performs quite lower for individual signals than a fused signal. SVM needs multi-sensor fusion strategy to improve fault identification capability. Whereas PSVM performs better for both the unfused individual signals as well as fused signals. PSVM with individual vibration or acoustic emission signals is good enough to automate the gearbox fault diagnosis process. If the complexity of the problem is higher, PSVM with fused vibration and acoustic emission signals is the best candidate for fault diagnosis of the synchromesh gearbox.

\section{FUNDING}

This work was supported by the Defence Research and Development Organisation (DRDO) - India

\section{REFERENCES}

Abidi, M. A., (1992). Fusion of multi dimensional data using regularization.Data Fusion in Robotics and Machine Intelligence, pp. 415-455.

AshkanMoosavian,

MeghdadKhazaee, GholamhassanNajafi, Maurice Kettner\&RizalmanMamat.(2015). Spark plug fault recognition based on sensor fusion and classifier combination using dempster-Shafer evidence theory.Applied Acoustics, vol. 93, pp. 120-129.doi: 10.1016/j.apacoust.2015.01.008

BostjanDolenc, PavleBoskoski\&DaniJurici. (2016). Distributed bearing fault diagnosis based on vibration analysis. Mechanical Systems and Signal Processing, vol. 66-67, pp. 521-532.doi: 10.1016/j.ymssp.2015.06.007

Bo-Suk Yang \&Kwang Jin Kim.(2006). Application of dempster-Shafer theory in fault diagnosis of induction motors using vibration and current signals.Mechanical Systems and Signal Processing, vol. 20, pp. 403420.doi: 10.1016/j.ymssp.2004.10.010

David Logan\&Joseph Mathew. (1996). Using the correlation dimension for vibration fault diagnosis of rolling element bearings-I. basic concepts. Mechanical Systems and Signal Processing, vol. 10(3), pp. 241-250.doi: 10.1006/mssp.1996.0018

FarisElasha, Matthew Greaves, David Mba\&AbdulmajidAddali. (2015). Application of acoustic emission in diagnostic of bearing faults within a helicopter gearbox.Procedia CIRP, vol. 38, pp. 3036.doi:

Gang Niu, Tian Han, Bo-Suk Yang \& Andy Chit Chiow Tan. (2007). Multi-agent decision fusion for motor fault diagnosis.Mechanical Systems and Signal Processing, vol. 21, pp. 1285-1299.doi: 10.1016/j.procir.2015.08.042

Hong Fei Wang\&Jiang Ping Wang. (2000). Fault diagnosis theory: method and application based on multisensor 
data fusion. Journal of Testing and Evaluation, vol. 28(6), pp. 513-518.doi: 10.1520/JTE12143J.

Hung-Chih Chiang, Randolph L. Moses \& Lee C. Potter. (2001). Model- based Bayesian feature matching with application to synthetic aperture radar target recognition. Pattern Recognition, vol. 34, pp. 15391553.doi: 10.1016/S0031-3203(00)00089-3

Jian Yang, Jing-Yu Yang, David Zhang \&Jian-Feng Lu. (2003). Feature fusion: parallel strategy vs. serial strategy. Pattern Recognition, vol. 36, pp. 13691381.doi: 10.1016/S0031-3203(02)00262-5

Junyan Yang, Youyun Zhang \& Yong Sheng Zhu.(2007). Intelligent fault diagnosis of rolling element bearing based on SVMS and fractal dimension.Mechanical Systems and Signal Processing, vol. 21, pp. 20122024.doi: 10.1016/j.ymssp.2006.10.005

Kuan Fang He, Jigang Wu \&Guang Bin Wang. (2012). Acoustic emission signal feature extraction in rotor crack fault diagnosis. Journal of Computers, vol. 7(9), pp. 2120-2127.

Lei, Y., Lin, J., He, Z., \&Zuo, M. J. (2013).A review on empirical mode decomposition in fault diagnosis of rotating machinery. Mechanical Systems and Signal Processing, vol. 35, pp. 108-126.doi: 10.1016/j.ymssp.2012.09.015

Li, N., Zhou, R., Hu, Q., \& Liu, X. (2012). Mechanical fault diagnosis based on redundant second-generation wavelet packet transform, neighborhood roughest and support vector machine, Mechanical Systems and Signal Processing, vol. 28, pp. 608-621. DOI: 10.1016/j.ymssp.2011.10.016

Loutas, T. H., Roulias, D., Pauly, E. \&Kostopoulos, V. (2011).The combined use of vibration, acoustic emission, and oil debris on-line monitoring towards a more effective condition monitoring of rotating machinery. Mechanical Systems and Signal Processing, vol. $25, \quad$ pp. 1339-1352.doi: 10.1016/j.ymssp.2010.11.007

MeghdadKhazaee, HojatAhmadi, Mahmoud Omid, AshkanMoosavian\&MajidKhazaee.(2014). Classifier fusion of vibration and acoustic signals for fault diagnosis and classification of planetary gears based on dempster-Shafer evidence theory.ProcIMech E Part E: $J$ Process Mechanical Engineering, vol. 228(1), pp. 2132.doi: $10.1177 / 0954408912469902$

OtmanBasir\& Xiao hong Yuan.(2007). Engine fault diagnosis based on multi-sensor information fusion using dempster-Shafer evidence theory.Information Fusion, vol. 8, pp. 379-386.doi: 10.1016/j.inffus.2005.07.003

Praveenkumar, T., Sabhrish, B., Saimurugan, M., \&Ramachandran, K.I. (2018).Pattern recognition based on-line vibration monitoring system for fault diagnosis of automobile gearbox. Measurement, vol. 114, 233242.doi: 10.1016/j.measurement.2017.09.041
Rai, V. K., \&Mohanty, A. R. (2007). Bearing fault diagnosis using FFT of intrinsic mode functions in Hilbert-Huang transform. Mechanical Systems and Signal Processing, vol. 21(8), pp. 3030-3041.doi: 10.1016/j.ymssp.2006.12.004

Rubini, R. \&Meneghetti, U. (2001). Application of the envelope and wavelet transform analyses for the diagnosis of incipient faults in ball bearings. Mechanical Systems and Signal Processing, vol. 15, pp. 287-302.doi: 10.1006/mssp.2000.1330

Chen, W., (1991). Nonlinear Analysis of Electronic Prognostics.Doctoral dissertation.The Technical University of Napoli, Napoli, Italy.

Ruoyu Li., (2012). Rotating Machine Fault Diagnostics using Vibration and Acoustic Emission Sensors. Doctoral dissertation.UIC Graduate College University of Illinois, Chicago.

Safizadeh, M. S., \&Latifi, S. K. (2014).Using multi-sensor data fusion for vibration fault diagnosis of rolling element bearings by accelerometer and load cell.Information Fusion, vol. 18, pp. 1-8.doi: 10.1016/j.inffus.2013.10.002

Saimurugan, M., \&Nithesh, R. (2016). Intelligent fault diagnosis model for rotating machinery based on fusion of sound signals, International Journal of Prognostics And Health Management, pp. 1-10.

Saimurugan, M., Ramachandran, K. I., Sugumaran, V., \&Sakthivel, N. R. (2011).Multi-component fault diagnosis of rotational mechanical system based on decision tree and support vector machine.Expert Systems with Applications, vol. 38, pp. 38193826.doi:10.1016/j.eswa.2010.09.042

Saimurugan, M., \& Ramprasad, R. (2017). A dual-sensor signal fusion approach for detection of faults in rotating machines. Journal of Vibration and Control, pp. 26212630.doi:10.1177/1077546316689644

Samanta, B., Al-Balushi, K. R., \& Al-Araimi, S. A. (2003). Artificial neural networks and support vector machines with genetic algorithm for bearing fault detection. Engineering Applications of Artificial Intelligence, vol. 16, pp. 657-665.doi:10.1016/j.engappai.2003.09.006

Sanjay Taneja. (2013). Effect of unbalance on performance of centrifugal pump.International Journal of Scientific \& Technology Research, vol. 2(8), pp. 56-60.

Saravanan, N., Kumar Siddabattuni, V. N. S., \&Ramachandran, K. I. (2010). Fault diagnosis of spur bevel gear box using artificial neural network (ANN), and proximal support vector machine (PSVM). Applied Soft Computing, vol. 10,pp.344360.doi:10.1016/j.asoc.2009.08.006

Sultan Binsaeid, ShihabAsfour, Sohyung Cho \&ArzuOnar.(2009). Machine ensemble approach for simultaneous detection of transient and gradual abnormalities in end milling using multisensor fusion.Journal of Materials Processing Technology, 
vol. 209, 2 pp. 4738.doi:10.1016/j.jmatprotec.2008.11.038

Wei Li, Zhencai Zhu, Fan Jiang, Gong Bo Zhou \&Guoan Chen. (2015). Fault diagnosis of rotating machinery with a novel statistical feature extraction and evaluation method.Mechanical Systems and Signal Processing, vol. 50-51, $\quad$ pp. 414426.doi:10.1016/j.ymssp.2014.05.034

Xiao Li Zhang, BaoJian Wang \&XueFeng Chen. (2015). Intelligent fault diagnosis of roller bearings with multivariable ensemble-based incremental support vector machine.Knowledge-Based Systems, vol. 89, pp. 56-85.doi:

Yang, Y., Yu, D. J., \& Cheng, J. S. (2007). A fault diagnosis approach for roller bearing based on IMF envelope spectrum and SVM.Measurement, vol. 40(910), pp. 943-950.doi:10.1016/j.knosys.2015.06.017

YongzhiQu, David He, Jae Yoon, Brandon Van Hecke, Eric Bechhoefer\&Junda Zhu. (2014). Gearbox tooth cut fault diagnostics using acoustic emission and vibration sensors - a comparative study. Sensors, vol. 14, pp. 1372-1393.doi: 10.3390/s140101372.

\section{BIOGRAPHIES}

Dr. T. Praveenkumar was born in the year 1990. He received his B.Tech degree in Automobile Engineering from SRM Institute of Science and Technology, Chennai, in the year 2011. He received his M.Tech degree in Automotive Engineering from Amrita School of Engineering, Amrita Vishwa Vidyapeetham, Coimbatore, in 2013. He received his Ph.D. from Amrita School of Engineering, Amrita Vishwa Vidyapeetham, Coimbatore, in 2019. He currently serves as Assistant Professor at Department of Automobile Engineering, SRM Institute of Science and Technology, Chennai. His areas of research include Automotive Transmission, Machine Learning, and Machine Condition Monitoring.

Dr. M. Saimurugan received his B.E degree in Mechanical Engineering from Kongu Engineering College, Bharathiar University, Coimbatore, in 1998. He received his M. E. degree in Computer-Aided Design from Government College of Engineering, Periyar University, Salem, in 2000. He received his Ph.D. from Amrita School of Engineering, Amrita Vishwa Vidyapeetham, Coimbatore, in 2013. He currently serves as Associate Professor at Department of Mechanical Engineering, Amrita School of Engineering, Coimbatore Campus. His areas of research include Vibration Analysis, Machine Learning, and Machine Condition Monitoring.

Dr. K. I. Ramachandran currently serves as Professor at Department of Mechanical Engineering and Professor at Center for Computational Engineering and Networking (CEN), Amrita School of Engineering, Amrita Vishwa Vidyapeetham University, Coimbatore Campus. His research interest includes: Computational Methods for Solids \& Fluids, Vibration Analysis, Monitoring \& Control, Fault Diagnosis \& Machine Learning, Molecular Simulation, Biomechanics, Biomedical Signal \& Image Processing. 